\title{
Proceeding
}

Supplementary Issue: Winter Conferences of Sports Science. Costa Blanca Sports Science Events, 22-23 March 2021. Alicante, Spain.

\section{Effects of motor and cognitive loads on postural stability in healthy children}

\author{
MARIANNA LIPAROTI \\ Department of Motor Sciences and Wellness, University of Naples "Parthenope", Italy
}

\begin{abstract}
Traditionally postural control was considered an automatic task, currently it is considered a process that involve higher control levels. The aims of study were to investigate the effects of cognitive and motor secondary tasks on postural control in healthy students and reveal which of two tasks have a greater impact on simple task. We enrolled eleven healthy children, who were asked to perform the following task: standing with open eyes and performing the same task while performing a cognitive (an n-back subtraction) or motor (finger tapping) dual task (DT). Our results demonstrated that both DT affects the postural control. Specifically, we find higher Centre of Pressure (COP) sway area during cognitive DT as compared to both single task and motor DT. Furthermore, results showed higher COP oscillation velocity in anteroposterior direction during cognitive DT then motor DT. Moreover, this parameter was significantly different in single task compared to motor DT. However, the secondary cognitive task showed a greater impact on postural control then motor secondary task on postural control. Our findings may be applied in school setting to elaborate motor programs useful to improve the postural stability and the ability to perform two tasks simultaneously.
\end{abstract}

Keywords: Postural stability; Dual task; Educational setting.

Cite this article as:

Liparoti, M. (2021). Effects of motor and cognitive loads on postural stability in healthy children. Journal of Human

Sport and Exercise, 16(3proc), S913-S921. https://doi.org/10.14198/ihse.2021.16.Proc3.08

Corresponding author. Department of Motor Sciences and Wellness, University of Naples "Parthenope", Italy. https://orcid.org/0000-0003-2192-6841

E-mail: marianna.liparoti@uniparthenope.it

Abstract submitted to: Winter Conferences of Sports Science. Costa Blanca Sports Science Events, 22-23 March 2021. Alicante, Spain.

JOURNAL OF HUMAN SPORT \& EXERCISE ISSN 1988-5202.

(c) Faculty of Education. University of Alicante.

doi:10.14198/jhse.2021.16.Proc3.08 


\section{INTRODUCTION}

In school setting the attentional deficits are a crucial topic, frequent lapses in attention, recurrent postural changes and restlessness are well known. Several factors could affect the attention, for example, when students perform two tasks simultaneously, the attention requests could compete and limited attention span may contribute in performing one or both tasks less efficiently (Woollacott and Shumway-Cook, 2002; YogevSeligmann et al., 2008). It may be important to understand which factors compete for attentional resources while students are performing different tasks, in order to create an environmental school more conducive to learning (Raiola et al., 2014; Raiola and Tafuri, 2015).

Attention is a cognitive process defined as the information processing capacity (Yogev-Seligmann et al., 2008). According to Kahneman's model, this process has a limited capacity and the execution of a tasks requires a portion of that capacity (Kahneman, 1973).

Traditionally, postural control was considered an automatic and reflex-dependent task, suggesting that postural control systems use higher attentional minimal resources. However in recent decades, scientific studies have shown that postural stability is a complex skill based on the interaction of dynamic sensorymotor processes that develop on different hierarchical levels, higher "controlled" and lower "automatic" levels of processing (Jacobs and Horak, 2007). Evidence showing the involvement of higher control levels in balance is based on studies that have demonstrated the theory of movement-specific reinvestment (Masters and Maxwell, 2008), in which conscious processing of movement while maintaining a posture has been manipulated. Masters et al. have observed that a reduction in superior control towards postural control, which can occur when attention is directed internally, could increase the probability to interrupt the automated motor execution. When the attention is focused internally, self-regulation mechanisms are triggered through which the performer assesses whether his movement corresponds to the performance that has as its objective. If the execution of the movement corresponds to the objective, the self-regulation mechanisms tend to decrease, while if there is no correspondence the subject tends to increase the requests for internal attention to reduce this discrepancy. Therefore, a specific reinvestment of movement which corresponds to a reinvestment of actions and perception with attention, could interrupt the automation of movement. The involvement of the higher control levels in postural control therefore falls within the theoretical framework of learning skills proposed by Fitts and Posner (Fitts and Posner, 1967). The involvement of higher or lower control levels depends on the characteristics of the postural task, the age of the individual, the ability to balance and the environment in which the subject moves.

In recent years, the relationship between cognition and movement has been investigated through the dual task paradigm (Woollacott and Shumway-Cook, 2002; Yogev-Seligmann et al., 2008). This paradigm provides for the simultaneous execution of two tasks in order to direct the attention of the performer to an external source of attention (e.g., reciting the letters of the alphabet, counting backwards) while performing a main task. In accordance with the hypothesis of constrained action (McNevin et al., 2003), a dual task condition could result in a shift of the attention focus from the intern to extern, improving motor performance. However, increased task complexity requires increased cognitive processing, and central interference could adversely affect performance. The dual task effects are investigated in various populations including, people with neurodegenerative disease (e.g. Parkinson, Alzheimer and Multiple sclerosis) (Jacini et al., 2018; Liparoti et al., 2019; Rucco et al., 2017), as well as both young and older healthy adult (Gobbo et al., 2014; Montuori et al., 2019; Raiola et al., 2015). Several studies about the dual task performance in healthy adult have reported that the performance of the secondary task negatively affects motor ability. For example Kerr et al. have been investigated the effects of attentional demands and the postural control in static condition in 
young adult comparing the spatial memory task respect to verbal memory task and they demonstrated that the performance of a memory task with the concurrent balance task caused an increase of error numbers in the spatial task (Kerr et al., 1985). However, in literature, studies about the effects of dual task paradigm focused on the use of only a low cognitive demand dual task and the use of complex cognitive task or motor task often it is not contemplated. Moreover, these effects are often investigated through the detection of reaction times and although this is an efficient technique, it does not take into account the variations of movement during the execution of the task. To overcome this problem, a biomechanical investigation could be useful to quantify the effect of cognitive-motor interference on a main task such as the ability to maintain postural stability in static conditions.

The 3D-Motion Analysis (3D-MA) techniques may be a suitable tools to study posture and how it changes during different tasks providing quantitative measurements of spatiotemporal parameters (Troisi Lopez et al., 2021). From biomechanical point of view, static balance is defined through the analysis of centre of mass (COM) movements in relation to the base of support (BOS) (Lugade et al., 2011). Precisely the centre of gravity (COG), that represent the projection of COM to the ground, should stay within the boundaries of BOS in order to keep the balance. This static balance occurs when the displacement of the centre of pressure (COP) through the feet is controlled and the body moves as a single segment like an inverted pendulum (Rucco et al., 2018). Previous studies have investigated the impact of the difference in position between the COP and COG on postural stability in static balance and it has been shown that a larger distance between COG and COP indicate a greater body acceleration during quiet standing.

The present study was carried out in order to assess the effects of cognitive-motor dual task paradigm on the ability to maintain the balance in static condition in healthy children students. To this aim we performed a 3D-MA in a cohort of healthy children students under different task conditions performed while the participants tried to maintain balance.

\section{METHODS}

\section{Participants}

Eleven healthy children students (nine males and two females) were recruited according to the following exclusion criteria: (i) muscular or skeletal dysfunctions; (ii) neurological diseases; (ii) obesity. Our cohort showed the following mean value of demographic and anthropometrics features: the age of 7.09 years \pm 2.38, the weight of $32.5 \pm 1.8$ and the height of $132.1 \pm 3.2$.

\section{Motion analysis evaluation}

The motion analysis assessment was performed in the Motion Analysis Laboratory of the University of Naples "Parthenope". The evaluation was carried out by using a Stereophotogrammetric system equipped by 8 infrared cameras (ProReflex Unit-Qualisys Inc., Gothenburg, Sweden, with a frequency sampling of $120 \mathrm{~Hz}$ ) and at the same time information relating to ground reaction forces was collected by a force platform (Kistler 9260AA) (Liparoti et al., 2020; Rucco et al., 2020; Sorrentino et al., 2016). Fifty-five passive markers were applied to each participant according to the modified Davis protocol on anatomical landmarks of feet, lower limbs joints, pelvis, trunk, upper limb joints and head (Davis et al., 1991). The recorded data were processed using a tracking data software (Qualisys Track Manager by Qualisys AB, Goteborg, Sweden) and a software (Visual 3D by C-Motion Inc., Germantown, MD) to rebuild and model the skeleton. The 3D-MA and the force platform were used to obtain the following parameters: COP area (area in $\mathrm{mm}^{2}$ of the ellipse that includes all the points of the COP measured and reported on a cartesian axis system), COG area (area in $\mathrm{mm}^{2}$ of the ellipse that includes all the points of the COG measured and reported on a cartesian axis system), average 
distance from ideal centre $X$ (it is the distance in millimetres on the $X$ axis (mediolateral axis) of the average COG compared to the ideal COG), average distance from ideal centre $Y$ (it is the distance in millimetres on the $Y$ axis (anteroposterior axis) of the average COG compared to the ideal COG), Velmax COGml \& ap (maximum velocity of displacement of the COG average expressed in millimetres per second on the mediolateral and anteroposterior directions), Velmax $\mathrm{COP}_{\mathrm{ml}}$ ap (maximum velocity of displacement of the COP average expressed in millimetres per second on the mediolateral and anteroposterior directions.

\section{Experimental protocol}

Participants were asked to remain in upright position while observing a fixed point on the wall (open eyes, $\mathrm{OE}$ ). Subsequently were asked to perform two tasks, proposed by the operator at random order: motor dual task (MotDT, consisting of the finger tapping task, in which the thumb makes contact with other four fingers of the dominant hand while they stood in an orthostatic position) and cognitive dual task (CogDT, counting backward aloud by $7 \mathrm{~s}$ from 100 while they stood in an orthostatic position). We recorded at least four trials for each task condition.

\section{Statistical analysis}

Statistical analysis was performed using MATLAB (MathWorks $®$, version R2013a). The means and standard deviations of the outcome measures were calculated. We computed the dual task cost (DTC) using the previously published equation (Liparoti et al., 2019), where OE was the basic task output and DT was a dual task output for a specific variable:

$$
D T=\frac{O E-D T}{O E} \times 100
$$

The normal distribution of variables was checked through the Shapiro-Wilk test. In order to compare, in all task conditions, the 3D-MA parameters we used the Friedman test. Subsequently, the post-hoc analysis was carried out using Wilcoxon test. The same test was used to estimate the cost of the dual task. The statistical significance was defined as $p<.05$.

\section{RESULTS}

The statistical analysis showed significant different in two motion parameters, the COP area $\left(X^{2}(\mathrm{df}=2, \mathrm{~N}=\right.$ $11)=3.45, p=.008)$ and the maximum velocity of COP anteroposterior $\left(X^{2}(d f=2, N=11)=6.73, p=.001\right)$ direction (Figure $1 \mathrm{~A}$ and $\mathrm{B}$ ). In detail, the post-hoc analysis showed significantly higher COP area during CogDT as compared to both OE $(p=.013)$ and MotDT $(p=.002)$. Furthermore, significantly higher maximum velocity of COP in anteroposterior direction was exhibited in CogDT as compared to both OE $(p=.041)$ and $\operatorname{MotDT}(p=.001)$. In addition, also significant difference was showed between OE and MotDT $(p=.001)$ in velocity of $\mathrm{COP}$ in anteroposterior direction.

Finally, we compared the impact of DT on postural control (Figure $1 \mathrm{C}$ ) and we found that the CogDT condition produced more consistent cost $(p=.039)$ then MotDT.

\section{DISCUSSION}

This investigation aimed to detect the effects of interference attention, induced by the execution of two different secondary tasks, motor and cognitive dual tasks, on postural control in children students. Furthermore, we estimate which of the two secondary tasks had a greater impact on posture. 
We find higher oscillation COP area during cognitive secondary task as compared to both single and motor secondary tasks. Our results also showed greater COP oscillation velocity in anteroposterior direction during cognitive dual task compared to motor secondary task.
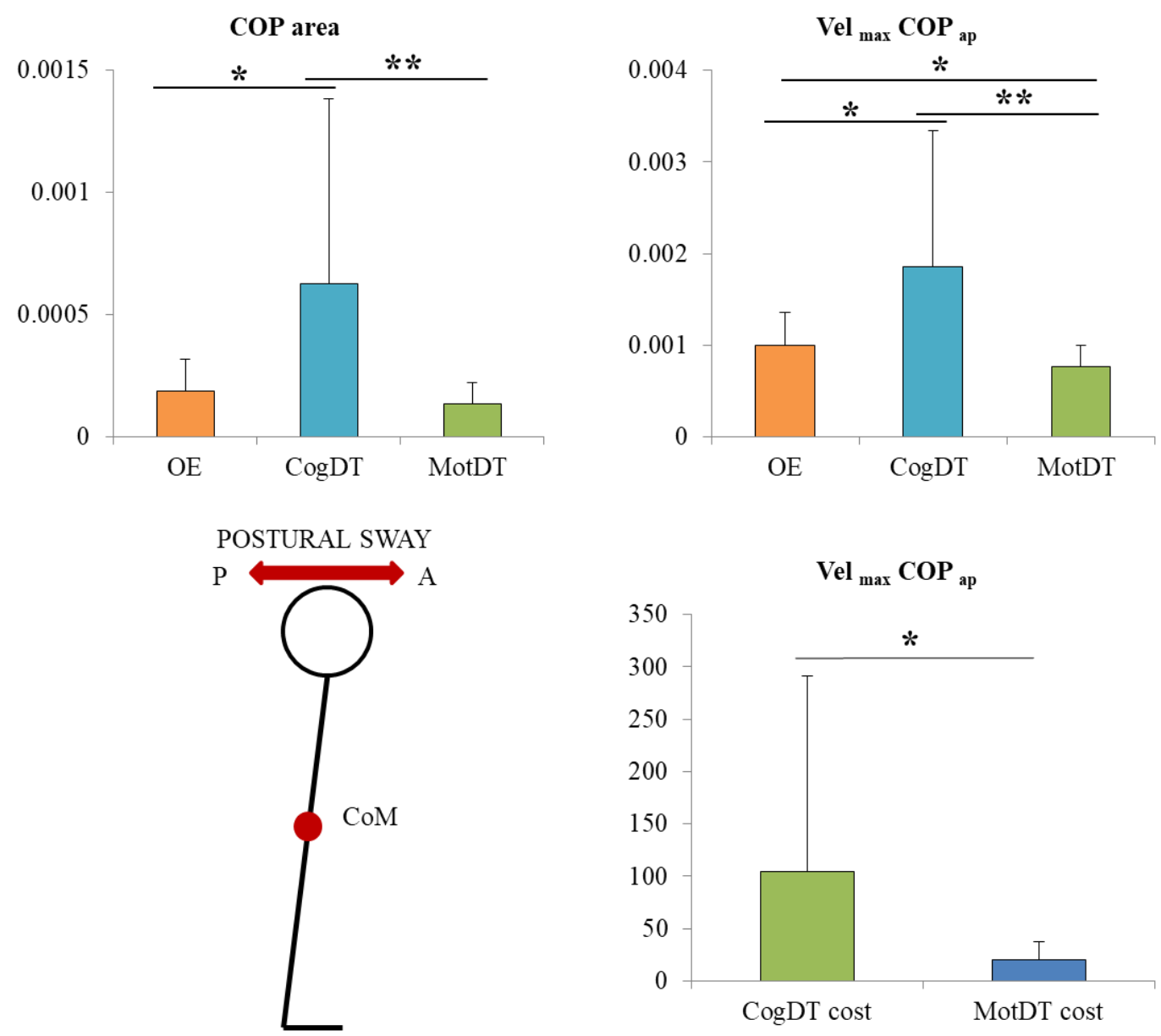

Plots of the centre of pressure (COP) during single-task (OE), cognitive dual task (CogDT) and motor dual task (MotDT) conditions in children students. (A) Graph show higher COP area during CogDT respect to both OE and MotDT. (B) Graphs illustrate higher maximum velocity of COP in anteroposterior direction during $\operatorname{Cog} D T$ then both OE and MotDT. In addition, show significant difference between OE and MotDT. C) Graphs illustrate higher impact of CogDT respect to MotDT in maximum velocity of COP in anteroposterior direction.

Figure 1. Postural control assessment.

Maintaining postural control while standing is often considered an automatic task, requiring minimal attention involvement and poor cognitive resources. However, even an automatic task when performed at the same time as a cognitive or motor task can be compromised and cause loss of balance and control. Previous researchers have studied the dual-task effects on postural control (Maylor and Wing, 1996; Rankin et al., 2000; Shumway-Cook and Woollacott, 2000), however some of them have only evaluated the effects of different cognitive dual task in different groups of participants and have not evaluating the effects of different types of tasks, such as motor secondary task. At the same time, several researchers did not check the secondary task load on the basic performance. In our study, we attempted to fill the gap of previous studies, using two different types of dual tasks, cognitive and motor secondary tasks, in order to understand the 
effects of a greater demand for brain resources when participants perform different types of secondary task and understand the degree of difficulty performing two tasks simultaneously.

In our study we have chosen a counting back task and a finger tapping task. The $n$-back task requires the working memory involvement that is defined as an integral component of executive functions engaged in complex cognitive coordination useful for the activity of daily living, since it allows to shifting attention from one task to another and perform two tasks simultaneously. Working memory is a skill that develops linearly during childhood and, depending on the maturation of the brain components, it reaches the adult level during adolescence (Gómez et al., 2018). For this reason, we thought it was interesting to study the effects of a secondary task that requires the involvement of working memory on postural control and to understand the strategies necessary to maintain balance. Instead, the finger tapping task is a simple motor task that involve the motor fatigue and motor learning. This task is useful to evaluate the rhythmic movement pattern and it is commonly used in clinical assessment because it is sensitive to the rhythmic movement alteration. In particular, this task includes repetitive and sequential movements representing respectively basic movements that develop early and movements that require higher order skills in later development.

Our results revealed the difficulty to allocate an adequate attention and brain resources when the participant perform a secondary task while maintaining the standing position, resulting in an unstable equilibrium as demonstrated by higher total displacement of COP during secondary cognitive task then other conditions. Although this result is interesting, it provides only a quantitative indication of the total COP shift and does not provide specific indications about mediolateral or anteroposterior body shifts or information on the strategy implemented to maintain balance and better manage cognitive load. To overcome this limit, we also calculated the postural sway variations in mediolateral and anteroposterior directions in the three experimental conditions (open eyes, cognitive and motor dual tasks). Our results revealed that the maximum velocity of COP displacement in anteroposterior direction was affected by both cognitive and mo tor secondary dual tasks compared to single task. Furthermore, the maximum velocity of COP displacement in anteroposterior direction was higher in cognitive dual task then in motor secondary task. This means that participants presented more body mass sway in AP direction during CogDT. This results are partially in accordance with previous study in which it was observed that the children have a greater body sway in AP direction than young children (Cherng et al., 2003). Our results may be explained by the body morphology of our cohort that may have more mass in upper extremity of body hence the inverted pendulum showed higher frequency sway in AP direction, furthermore the body stability is still developing. In summary we have shown that postural control during standing is affected by a secondary task regardless of the type of task being performed, therefore the body is predisposed to a risk of loss of balance. Attentional resources are limited when shared between primary and secondary tasks and a competition of brain resources leads to a deterioration in performance, known as dual-task cost.

The dual task cost express the impact of secondary task on primary task (Siu and Woollacott, 2007; Woollacott and Shumway-Cook, 2002). We calculate the cost of dual task and our results showed higher cognitive dual task impact in the maximum velocity of COP displacement in anteroposterior direction then motor dual task. This result highlight that the type of secondary task is one important factor which can impact dual task performance. During cognitive dual task condition the level of difficulty is higher and there is a decrease in additional information that can be retained and less efficient motor performance when two tasks are performed separately. The complexity of the task have a pivotal role in the success of the performance. The more cognitive resources are needed, the lower the efficiency of primary performance. The interference of postural control in the present study could result from immaturity of integrative sensory functions and a reduced ability to control posture when several stimuli were processed at the same time. With advancing of 
age, integrative sensory functions develop and the ability to manage two tasks simultaneously could improve the control of posture stability during dual task paradigm.

This is a preliminary study and has a number of limitations that may be addressed in future studies. For example, we evaluated only one type of cognitive task and only one type of motor task it would be interesting to observe how tasks of different difficulty and requiring the use of different cognitive resources can affect the ability to control posture. It would also be interesting to observe how this ability evolves with increasing age and therefore with a greater ability to manage two tasks simultaneously.

\section{Application of research in educational setting}

In our study, we demonstrated that there is a relationship between cognition and movement control. Specifically, the students experienced the interference of a dual cognitive and motor task with respect to a simple main task such as standing. We observed the inability to control posture, which presented with higher maximum displacement of the COP in the anteroposterior direction during the execution of the dual cognitive task.

In the academic field, this type of information could be very useful for planning experiences and creating a more conducive learning environment (Gaetano et al., 2015; Sorrentino et al., 2019). Performing a cognitive task that requires attention could overload naturally limited attentional resources, resulting in interference of postural control and loss of balance. For example, experiences of exploration and discovery that are accomplished, using the various parts of the body, playing and manipulating objects, through which the gradual construction of the body scheme is favoured, intended as a representation of the image of the body in globally or multilinked segments. Activities that require not only motor but also cognitive involvement could be integrated into the school program, for example meditation is a mind-body practice more and more in different educational and training contexts and which improves not only an improvement in movement but also functions cognitive (Lardone et al., 2018; Minino et al., 2020). Furthermore, the results of this study support the need to include, in academic contexts, motor education programs useful for the development of motor control based on perceptual-motor activities, that takes into account the needs of all children, including children who require special and personalized attention, such as children with Down syndrome, autism or spastic paraplegia (Rucco et al., 2019).

The more automatic postural control, less attentional resources needed to postural control and therefore more are the attentional resources should be available for cognitive processing.

\section{CONCLUSION}

In conclusion, our results show that postural control cannot be considered just an automatic task but there is a strong relationship between cognition and movement. In particular, a dual task performance can affect the ability to maintain the standing position, depending on the secondary activity required. Our results highlight the importance of developing new motor education programs in order to make posture control more automatic and improve the ability to manage tasks performed simultaneously.

\section{REFERENCES}

Cherng, R.J., Lee, H.Y., Su, F.C., 2003. Frequency spectral characteristics of standing balance in children and young adults. Med. Eng. Phys. 25, 509-515. https://doi.org/10.1016/S13504533(03)00049-3 
Davis, R.B., Ounpuu, S., Tyburski, D., Gage, J.R., 1991. A gait analysis data collection and reduction technique. Hum. Mov. Sci. 10, 575-587. https://doi.org/10.1016/0167-9457(91)90046-Z

Fitts, P.M., Posner, M.I., 1967. Human performance.

Gaetano, R., Domenico, T., Gaetano, A., 2015. Physical activity and its relation to body and ludic expression in childhood. Mediterr. J. Soc. Sci. 6, 293. https://doi.org/10.5901/mjss.2015.v6n3s2p293

Gobbo, S., Bergamin, M., Sieverdes, J.C., Ermolao, A., Zaccaria, M., 2014. Effects of exercise on dualtask ability and balance in older adults: a systematic review. Arch. Gerontol. Geriatr. 58, 177-187. https://doi.org/10.1016/j.archger.2013.10.001

Gómez, C.M., Barriga-Paulino, C.I., Rodríguez-Martínez, E.I., Rojas-Benjumea, M.Á., Arjona, A., Gómez-González, J., 2018. The neurophysiology of working memory development: From childhood to adolescence and young adulthood. Rev. Neurosci. 29, 261-282. https://doi.org/10.1515/revneuro2017-0073

Jacini, F., Sorrentino, P., Lardone, A., Rucco, R., Baselice, F., Cavaliere, C., Aiello, M., Orsini, M., lavarone, A., Manzo, V., Carotenuto, A., Granata, C., Hillebrand, A., Sorrentino, G., 2018. Amnestic mild cognitive impairment is associated with frequency-specific brain network alterations in temporal poles. Front. Aging Neurosci. 10, 1-11. https://doi.org/10.3389/fnagi.2018.00400

Jacobs, J. V, Horak, F.B., 2007. Cortical control of postural responses. J. Neural Transm. 114, 13391348. https://doi.org/10.1007/s00702-007-0657-0

Kahneman, D., 1973. Attention and effort. Citeseer.

Kerr, B., Condon, S.M., McDonald, L.A., 1985. Cognitive spatial processing and the regulation of posture. J. Exp. Psychol. Hum. Percept. Perform. 11, 617. https://doi.org/10.1037/0096-1523.11.5.617

Lardone, A., Liparoti, M., Sorrentino, P., Rucco, R., Jacini, F., Polverino, A., Minino, R., Pesoli, M., Baselice, F., Sorriso, A., 2018. Mindfulness Meditation Is Related to Long-Lasting Changes in Hippocampal Functional Topology during Resting State: A Magnetoencephalography Study. Neural Plast. 2018. https://doi.org/10.1155/2018/5340717

Liparoti, M., Della Corte, M., Rucco, R., Sorrentino, P., Sparaco, M., Capuano, R., Minino, R., Lavorgna, L., Agosti, V., Sorrentino, G., 2019. Gait abnormalities in minimally disabled people with Multiple Sclerosis: A 3D-Motion Analysis study. Mult. Scler. Relat. Disord. https://doi.org/10.1016/i.msard.2019.01.028

Liparoti, M., Troisi Lopez, E., Agosti, V., 2020. Motion capture system: A useful tool to study cyclist's posture.

Lugade, V., Lin, V., Chou, L.-S., 2011. Center of mass and base of support interaction during gait. Gait Posture 33, 406-411. https://doi.org/10.1016/i.gaitpost.2010.12.013

Masters, R., Maxwell, J., 2008. The theory of reinvestment. Int. Rev. Sport Exerc. Psychol. 1, 160-183. https://doi.org/10.1080/17509840802287218

Maylor, E.A., Wing, A.M., 1996. Age differences in postural stability are increased by additional cognitive demands. Journals Gerontol. Ser. B Psychol. Sci. Soc. Sci. 51, P143-P154. https://doi.org/10.1093/geronb/51B.3.P143

McNevin, N.H., Shea, C.H., Wulf, G., 2003. Increasing the distance of an external focus of attention enhances learning. Psychol. Res. 67, 22-29. https://doi.org/10.1007/s00426-002-0093-6

Minino, R., Belfiore, P., Liparoti, M., 2020. Neuroplasticity and motor learning in sport activity. J. Phys. Educ. Sport 20.

Montuori, S., D'Aurizio, G., Foti, F., Liparoti, M., Lardone, A., Pesoli, M., Sorrentino, G., Mandolesi, L., Curcio, G., Sorrentino, P., 2019. Executive functioning profiles in elite volleyball athletes: Preliminary results by a sport-specific task switching protocol. Hum. Mov. Sci. 63, 73-81. https://doi.org/10.1016/j.humov.2018.11.011 
Raiola, G., Lipoma, M., Tafuri, D., 2015. Postural control in young soccer players: differences between the cognitive approach and ecological-dynamic one. https://doi.org/10.14198/jhse.2015.10.Proc1.29

Raiola, G., Tafuri, D., 2015. Teaching method of physical education and sports by prescriptive or heuristic learning. J. Hum. Sport Exerc. 10, S377-S384. https://doi.org/10.14198/jhse.2015.10.Proc1.28

Raiola, G., Tafuri, D., Gomez Paloma, F., 2014. Physical activity and sport skills and its relation to mind theory on motor control. Sport Sci. 7, 52-56.

Rankin, J.K., Woollacott, M.H., Shumway-Cook, A., Brown, L.A., 2000. Cognitive influence on postural stability: a neuromuscular analysis in young and older adults. Journals Gerontol. Ser. A Biol. Sci. Med. Sci. 55, M112-M119. https://doi.org/10.1093/gerona/55.3.M112

Rucco, R., Agosti, V., Jacini, F., Sorrentino, P., Varriale, P., De Stefano, M., Milan, G., Montella, P., Sorrentino, G., 2017. Spatio-temporal and kinematic gait analysis in patients with Frontotemporal dementia and Alzheimer's disease through 3D motion capture. Gait Posture 52, 312-317. https://doi.org/10.1016/..gaitpost.2016.12.021

Rucco, R., Liparoti, M., Agosti, V., 2020. A new technical method to analyse the kinematics of the human movements and sports gesture.

Rucco, R., Liparoti, M., Jacini, F., Baselice, F., Antenora, A., De Michele, G., Criscuolo, C., Vettoliere, A., Mandolesi, L., Sorrentino, G., Sorrentino, P., 2019. Mutations in the SPAST gene causing hereditary spastic paraplegia are related to global topological alterations in brain functional networks. Neurol. Sci. 40, 979-984. https://doi.org/10.1007/s10072-019-3725-y

Rucco, R., Sorriso, A., Liparoti, M., Ferraioli, G., Sorrentino, P., Ambrosanio, M., Baselice, F., 2018. Type and Location of Wearable Sensors for Monitoring Falls during Static and Dynamic Tasks in Healthy Elderly: A Review. Sensors 18, 1613. https://doi.org/10.3390/s18051613

Shumway-Cook, A., Woollacott, M., 2000. Attentional demands and postural control: the effect of sensory context. Journals Gerontol. Sci. Med. Sci. 55, M10.

Siu, K.-C., Woollacott, M.H., 2007. Attentional demands of postural control: the ability to selectively allocate information-processing resources. Gait Posture 25, 121-126. https://doi.org/10.1016/i.gaitpost.2006.02.002

Sorrentino, P., Barbato, A., Del Gaudio, L., Rucco, R., Varriale, P., Sibilio, M., Strazzullo, P., Sorrentino, G., Agosti, V., 2016. Impaired gait kinematics in type 1 Gaucher's Disease. J. Parkinsons. Dis. 6, 191-195. https://doi.org/10.3233/JPD-150660

Sorrentino, P., Lardone, A., Pesoli, M., Liparoti, M., Montuori, S., Curcio, G., Sorrentino, G., Mandolesi, L., Foti, F., 2019. The development of spatial memory analyzed by means of ecological walking task. Front. Psychol. 10. https://doi.org/10.3389/fpsyg.2019.00728

Troisi Lopez, E., Minino, R., Sorrentino, P., Rucco, R., Carotenuto, A., Agosti, V., Tafuri, D., Manzo, V., Liparoti, M., Sorrentino, G., 2021. A synthetic kinematic index of trunk displacement conveying the overall motor condition in Parkinson's disease. Sci. Rep. 11, 1-11. https://doi.org/10.1038/s41598021-82348-4

Woollacott, M., Shumway-Cook, A., 2002. Attention and the control of posture and gait: a review of an emerging area of research. Gait Posture 16, 1-14. https://doi.org/10.1016/S0966-6362(01)00156-4

Yogev-Seligmann, G., Hausdorff, J.M., Giladi, N., 2008. The role of executive function and attention in gait. Mov. Disord. 23, 329-342. https://doi.org/10.1002/mds.21720

\section{(2) $\odot \Theta \Theta$}

This work is licensed under a Attribution-NonCommercial-NoDerivatives 4.0 International (CC BY-NC-ND 4.0). 